\title{
Age-associated changes in podocytes linked to FGGS
}

Decreasing podocyte density with increasing age is associated with hypertrophic podocyte stress and can lead to glomerular tuft collapse and the development of focal global glomerulosclerosis (FGGS), say researchers. "This sequence of events provides a mechanism for the development of FGGS and partly explains why an increasing proportion of glomeruli become sclerotic as kidneys age, thereby contributing to ageassociated kidney failure," explains Roger Wiggins.

Kidney ageing is associated with a progressive decrease in kidney function. "Older age is recognized as the single most important factor associated with kidney failure, which is responsible for an unsustainable US\$40 billion in dialysis costs per year," says Wiggins. "The mechanisms for this association are not well understood but have been assumed to be largely related to atherosclerosis." According to Wiggins, analysis of biopsy samples from transplant recipients suggested an effect of ageing on podocyte density, as had previously been observed in rats. "It became clear that we needed to know what happened with age, hence we embarked on an effort to define how podocyte parameters change with age."

To study age-related changes in podocytes, Wiggins and colleagues developed a new technology they call 'podometrics.' "We developed this quantitative methodology because we realized from animal model studies that quantifying podocyte parameters was critical to understanding glomerular disease progression," says Wiggins. "The conventional stereometric methods are so time consuming that they are impractical for large-scale application."

The researchers measured glomerular volume and podocyte number, density and size in 89 normal kidney samples from living and deceased kidney donors and the normal poles of nephrectomies for cancer. They found a decrease in podocyte nuclear density of $\sim 0.9 \%$ per year from $>300$ per $10^{6} \mu \mathrm{m}^{3}$ at $<20$ years to $<100$ per $10^{6} \mu \mathrm{m}^{3}$ by $70-80$ years. This decrease in podocyte density with age was associated with an increase in glomerular tuft volume and a decrease in podocyte number per tuft, requiring a compensatory podocyte hypertrophic response. In kidneys from older individuals with low podocyte density, Wiggins and coworkers noted the appearance of protein in the Bowman space, together with evidence of binucleate podocytes, glomerular tuft collapse and FGGS. The researchers found similar pathologic changes in five biopsy samples from younger patients with underlying glomerular disease.

Wiggins believes that understanding glomerular disease progression in terms of decreased podocyte density—caused by increased glomerular volume and/or decreased podocyte number-offers new targets for therapeutic intervention, as well as tools for the clinician to monitor and prevent kidney failure.

Susan J. Allison

Original article Hodgin, J. B. et al. Glomerular aging and focal global glomerulosclerosis: a podometric perspective. J. Am. Soc. Nephrol. doi:10.1681/ASN.2014080752

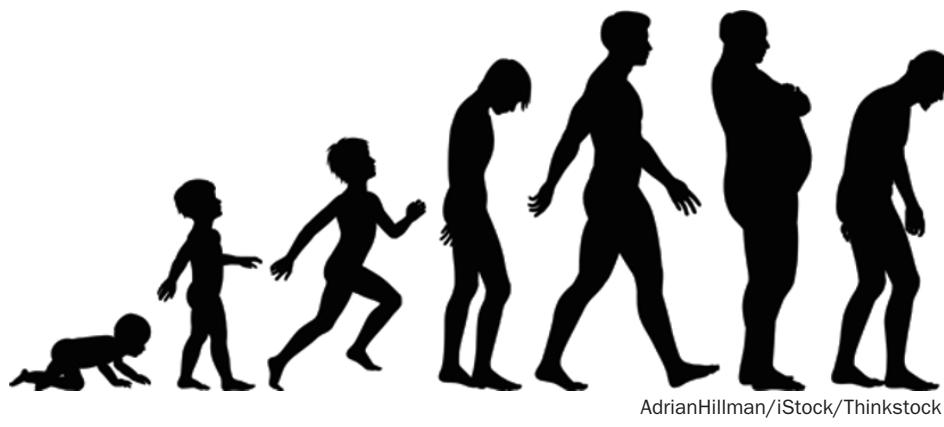

